Research Article

\title{
Generalizations on Some Hermite-Hadamard Type Inequalities for Differentiable Convex Functions with Applications to Weighted Means
}

\author{
Banyat Sroysang \\ Department of Mathematics and Statistics, Faculty of Science and Technology, Thammasat University, Pathum Thani 12121, Thailand \\ Correspondence should be addressed to Banyat Sroysang; banyat@mathstat.sci.tu.ac.th \\ Received 5 August 2013; Accepted 10 October 2013; Published 16 January 2014 \\ Academic Editors: J.-S. Chen and T. Li \\ Copyright (C) 2014 Banyat Sroysang. This is an open access article distributed under the Creative Commons Attribution License, \\ which permits unrestricted use, distribution, and reproduction in any medium, provided the original work is properly cited. \\ Some new Hermite-Hadamard type inequalities for differentiable convex functions were presented by Xi and Qi. In this paper, we \\ present new generalizations on the Xi-Qi inequalities.
}

\section{Introduction}

The Hermite-Hadamard inequality [1-3] states that if $f$ is a convex function on $[a, b]$, then

$$
f\left(\frac{a+b}{2}\right) \leq \frac{1}{b-a} \int_{a}^{b} f(x) d x \leq \frac{f(a)+f(b)}{2} .
$$

Let $f: I \subseteq \mathbb{R} \rightarrow \mathbb{R}$ be differentiable on $I^{\circ}$ and $\{a, b\} \subseteq I$ with $a<b$. Below we recall some Hermite-Hadamard type inequalities.

In 1998, Dragomir and Agarwal [4] showed that (i) if $\left|f^{\prime}(x)\right|$ is convex on $[a, b]$, then

$$
\begin{array}{r}
\left|\frac{f(a)+f(b)}{2}-\frac{1}{b-a} \int_{a}^{b} f(x) d x\right| \\
\leq \frac{(b-a)\left(\left|f^{\prime}(a)\right|+\left|f^{\prime}(b)\right|\right)}{8},
\end{array}
$$

and (ii) if $\left|f^{\prime}(x)\right|^{p /(p-1)}$ is convex on $[a, b]$ with $p>1$, then

$$
\begin{aligned}
& \left|\frac{f(a)+f(b)}{2}-\frac{1}{b-a} \int_{a}^{b} f(x) d x\right| \\
& \leq \frac{b-a}{2(p+1)^{1 / p}} \\
& \quad \times\left(\frac{\left|f^{\prime}(a)\right|^{p /(p-1)}+\left|f^{\prime}(b)\right|^{p /(p-1)}}{2}\right)^{(p-1) / p} .
\end{aligned}
$$

In 2000, Pearce and Pečarić [5] showed that if $\left|f^{\prime}(x)\right|^{q}$ is convex on $[a, b]$ with $q \geq 1$, then

$$
\begin{gathered}
\left|\frac{f(a)+f(b)}{2}-\frac{1}{b-a} \int_{a}^{b} f(x) d x\right| \\
\leq \frac{b-a}{4}\left(\frac{\left|f^{\prime}(a)\right|^{q}+\left|f^{\prime}(b)\right|^{q}}{2}\right)^{1 / q},
\end{gathered}
$$




$$
\begin{gathered}
\left|f\left(\frac{a+b}{2}\right)-\frac{1}{b-a} \int_{a}^{b} f(x) d x\right| \\
\leq \frac{b-a}{4}\left(\frac{\left|f^{\prime}(a)\right|^{q}+\left|f^{\prime}(b)\right|^{q}}{2}\right)^{1 / q} .
\end{gathered}
$$

In 2004, Kirmaci [6] showed that if $\left|f^{\prime}(x)\right|^{p /(p-1)}$ is convex on $[a, b]$ with $p>1$, then

$$
\begin{aligned}
& \left|f\left(\frac{a+b}{2}\right)-\frac{1}{b-a} \int_{a}^{b} f(x) d x\right| \\
& \quad \leq \frac{b-a}{4}\left(\frac{4}{p+1}\right)^{1 / p}\left(\left|f^{\prime}(a)\right|+\left|f^{\prime}(b)\right|\right), \\
& \left|f\left(\frac{a+b}{2}\right)-\frac{1}{b-a} \int_{a}^{b} f(x) d x\right| \\
& \leq \frac{b-a}{16}\left(\frac{4}{p+1}\right)^{1 / p} \\
& \quad \times\left(\left|f^{\prime}(a)\right|^{p /(p-1)}+3\left|f^{\prime}(b)\right|^{p /(p-1)}\right)^{(p-1) / p} \\
& \quad+\frac{b-a}{16}\left(\frac{4}{p+1}\right)^{1 / p} \\
& \quad \times\left(3\left|f^{\prime}(a)\right|^{p /(p-1)}+\left|f^{\prime}(b)\right|^{p /(p-1)}\right)^{(p-1) / p} .
\end{aligned}
$$

In 2010, Sarikaya et al. [7] showed that if $f^{\prime} \in L[a, b]$ and $\left|f^{\prime}(x)\right|^{q}$ is convex on $[a, b]$ with $q \geq 1$, then

$$
\begin{aligned}
& \left|\frac{1}{6}\left[f(a)+f(b)+4 f\left(\frac{a+b}{2}\right)\right]-\frac{1}{b-a} \int_{a}^{b} f(x) d x\right| \\
& \leq \frac{b-a}{12}\left(\frac{2^{q+1}+1}{3(q+1)}\right)^{1 / q} \\
& \times\left[\left(\frac{3\left|f^{\prime}(a)\right|^{q}+\left|f^{\prime}(b)\right|^{q}}{4}\right)^{1 / q}\right. \\
& \left.+\left(\frac{\left|f^{\prime}(a)\right|^{q}+3\left|f^{\prime}(b)\right|^{q}}{4}\right)^{1 / q}\right]
\end{aligned}
$$

$$
\begin{aligned}
& \left|\frac{1}{6}\left[f(a)+f(b)+4 f\left(\frac{a+b}{2}\right)\right]-\frac{1}{b-a} \int_{a}^{b} f(x) d x\right| \\
& \leq \frac{5(b-a)}{72} \\
& \times\left[\left(\frac{61\left|f^{\prime}(a)\right|^{q}+29\left|f^{\prime}(b)\right|^{q}}{90}\right)^{1 / q}\right. \\
& \left.\quad+\left(\frac{29\left|f^{\prime}(a)\right|^{q}+61\left|f^{\prime}(b)\right|^{q}}{90}\right)^{1 / q}\right] .
\end{aligned}
$$

In 2012, Xi and Qi [8] showed that if $\lambda, \mu \in[0,1]$ and if $f^{\prime} \in L[a, b]$ and $\left|f^{\prime}(x)\right|^{q}$ is convex on $[a, b]$ with $q \geq 1$, then

$$
\begin{aligned}
& \left|\frac{\lambda f(a)+\mu f(b)}{2}+\frac{2-\lambda-\mu}{2} f\left(\frac{a+b}{2}\right)-\frac{1}{b-a} \int_{a}^{b} f(x) d x\right| \\
& \leq \frac{b-a}{8}\left(1-2 \lambda+2 \lambda^{2}\right)^{1-1 / q} \\
& \times\left[\left(\left(4-9 \lambda+12 \lambda^{2}-2 \lambda^{3}\right)\left|f^{\prime}(a)\right|^{q}\right.\right. \\
& \left.\left.+\left(2-3 \lambda+2 \lambda^{3}\right)\left|f^{\prime}(b)\right|^{q}\right) \times(6)^{-1}\right]^{1 / q} \\
& +\frac{b-a}{8}\left(1-2 \mu+2 \mu^{2}\right)^{1-1 / q} \\
& \times\left[\left(\left(2-3 \mu+2 \mu^{3}\right)\left|f^{\prime}(a)\right|^{q}\right.\right. \\
& \left.\left.+\left(4-9 \mu+12 \mu^{2}-2 \mu^{3}\right)\left|f^{\prime}(b)\right|^{q}\right) \times(6)^{-1}\right]^{1 / q}, \\
& \left|\frac{\lambda f(a)+\mu f(b)}{2}+\frac{2-\lambda-\mu}{2} f\left(\frac{a+b}{2}\right)-\frac{1}{b-a} \int_{a}^{b} f(x) d x\right| \\
& \leq \frac{b-a}{4}\left(\frac{1}{2(q+1)(q+2)}\right)^{1 / q} \\
& \times\left[\left((q+3-\lambda)(1-\lambda)^{q+1}+(2 q+4-\lambda) \lambda^{q+1}\right)\left|f^{\prime}(a)\right|^{q}\right. \\
& \left.+\left((q+1+\lambda)(1-\lambda)^{q+1}+\lambda^{q+2}\right)\left|f^{\prime}(b)\right|^{q}\right]^{1 / q} \\
& +\frac{b-a}{4}\left(\frac{1}{2(q+1)(q+2)}\right)^{1 / q} \\
& \times\left[\left((q+1+\mu)(1-\mu)^{q+1}+\mu^{q+2}\right)\left|f^{\prime}(a)\right|^{q}\right. \\
& +\left((q+3-\mu)(1-\mu)^{q+1}+(2 q+4-\mu) \mu^{q+1}\right) \\
& \left.\times\left|f^{\prime}(b)\right|^{q}\right]^{1 / q} \text {. }
\end{aligned}
$$

Moreover, for other results involving the HermiteHadamard type inequalities, we also refer to [9-23].

In this paper, we generalize the Xi-Qi inequalities. 


\section{Preliminaries}

Lemma 1. Let $\lambda, \mu \in \mathbb{R}$ and let $f: I \subseteq \mathbb{R} \rightarrow \mathbb{R}$ be differentiable on $I^{\circ}$ and $\{a, b\} \subseteq I$ with $a<b$. Assume that $f^{\prime} \in$ $L[a, b]$ and $0<\epsilon<b-a$. Then

$$
\begin{aligned}
& \epsilon \lambda f(a)+(b-a-\epsilon) \mu f(b) \\
& +[\epsilon(1-\lambda)+(b-a-\epsilon)(1-\mu)] f(a+\epsilon)-\int_{a}^{b} f(x) d x \\
& =\int_{0}^{1}\left[\epsilon^{2}(1-\lambda-t) f^{\prime}(t a+(1-t)(a+\epsilon))\right. \\
& \left.\quad+(b-a-\epsilon)^{2}(\mu-t)\right] \\
& \quad \times f^{\prime}(t(a+\epsilon)+(1-t) b) d t .
\end{aligned}
$$

Proof. Integrating by part and changing variable, we have

$$
\begin{aligned}
& \int_{0}^{1} \epsilon^{2}(1-\lambda-t) f^{\prime}(t a+(1-t)(a+\epsilon)) d t \int_{t=0}^{t=1} \epsilon(\lambda+t-1) d f(t a+(1-t)(a+\epsilon)) \\
&= {[\epsilon(\lambda+t-1) f(t a+(1-t)(a+\epsilon))]_{t=0}^{t=1} } \\
&-\epsilon \int_{0}^{1} f(t a+(1-t)(a+\epsilon)) d t \\
&= \epsilon \lambda f(a)+\epsilon(1-\lambda) f(a+\epsilon)-\int_{a}^{a+\epsilon} f(x) d x, \\
& \int_{0}^{1} \quad(b-a-\epsilon)^{2}(\mu-t) f^{\prime}(t(a+\epsilon)+(1-t) b) d t \\
&=\int_{t=0}^{t=1}(b-a-\epsilon)(t-\mu) d f(t(a+\epsilon)+(1-t) b) \\
&= {[(b-a-\epsilon)(t-\mu) f(t(a+\epsilon)+(1-t) b)]_{t=0}^{t=1} } \\
&= \quad(b-a-\epsilon)(1-\mu) f(a+\epsilon) \\
&-(b-a-\epsilon) \int_{0}^{1} f(t(a+\epsilon)+(1-t) b) d t \\
& \\
&=(b-a-\epsilon) \mu f(b)-\int_{a+\epsilon}^{b} f(x) d x .
\end{aligned}
$$

Thus,

$$
\begin{aligned}
& \int_{0}^{1}\left[\epsilon^{2}(1-\lambda-t) f^{\prime}(t a+(1-t)(a+\epsilon))\right. \\
& \left.\quad+(b-a-\epsilon)^{2}(\mu-t)\right] f^{\prime}(t(a+\epsilon)+(1-t) b) d t \\
& =\epsilon \lambda f(a)+(b-a-\epsilon) \mu f(b) \\
& \quad+[\epsilon(1-\lambda)+(b-a-\epsilon)(1-\mu)] \\
& \quad \times f(a+\epsilon)-\int_{a}^{b} f(x) d x .
\end{aligned}
$$

Lemma 2 (see $[8])$. Let $s>0$ and $0 \leq \xi \leq 1$. Then

$$
\begin{gathered}
\int_{0}^{1}|\xi-t|^{s} d t=\frac{\xi^{s+1}+(1-\xi)^{s+1}}{s+1} \\
\int_{0}^{1} t|\xi-t|^{s} d t=\frac{\xi^{s+2}+(s+1+\xi)(1-\xi)^{s+1}}{(s+1)(s+2)} .
\end{gathered}
$$

\section{Main Results}

Theorem 3. Let $\lambda, \mu \in[0,1]$ and let $f: I \subseteq \mathbb{R} \rightarrow \mathbb{R}$ be differentiable on $I^{\circ}$ and $\{a, b\} \subseteq I$ with $a<b$. Assume that $f^{\prime} \in L[a, b]$ and $0<\epsilon<b-a$. If $\left|f^{\prime}(x)\right|^{q}$ is convex on $[a, b]$ with $q \geq 1$, then

$$
\begin{aligned}
& \mid \epsilon \lambda f(a)+(b-a-\epsilon) \mu f(b) \\
& +[\epsilon(1-\lambda)+(b-a-\epsilon)(1-\mu)] \\
& \times f(a+\epsilon)-\int_{a}^{b} f(x) d x \mid \leq \epsilon^{2}\left(\frac{(1-\lambda)^{2}+\lambda^{2}}{2}\right)^{1-(1 / q)} \\
& \times\left\{\frac{1}{6}\left[3-6 \lambda+6 \lambda^{2}-\frac{\epsilon}{b-a}\left(2-3 \lambda+2 \lambda^{3}\right)\right]\left|f^{\prime}(a)\right|^{q}\right. \\
& \left.+\quad+\frac{1}{6}\left[\frac{\epsilon}{b-a}\left(2-3 \lambda+2 \lambda^{3}\right)\right]\left|f^{\prime}(b)\right|^{q}\right\}^{1 / q} \\
& \times\left\{\frac{1}{6}\left[\left(1-\frac{\epsilon}{b-a}\right)\left(2-3 \mu+2 \mu^{3}\right)\right]\left|f^{\prime}(a)\right|^{q}\right. \\
& \left.\quad+\frac{1}{6}\left[1-3 \mu+6 \mu^{2}-2 \mu^{3}+\frac{\mu^{2}+(1-\mu)^{2}}{b-a}\right)^{1-(1 / q)}\left(2-3 \mu+2 \mu^{3}\right)\right] \\
& \left.\quad \times\left|f^{\prime}(b)\right|^{q}\right\}^{1 / q} \cdot
\end{aligned}
$$

Proof. Suppose that $\left|f^{\prime}(x)\right|^{q}$ is convex on $[a, b]$ with $q \geq 1$. By Lemma 1, we have

$$
\begin{aligned}
& \mid \epsilon \lambda f(a)+(b-a-\epsilon) \mu f(b) \\
& +[\epsilon(1-\lambda)+(b-a-\epsilon)(1-\mu)] \\
& \times f(a+\epsilon)-\int_{a}^{b} f(x) d x\left|\leq \epsilon^{2} \int_{0}^{1}\right| 1-\lambda-t \mid \\
& \quad \times\left|f^{\prime}(t a+(1-t)(a+\epsilon))\right| d t \\
& +(b-a-\epsilon)^{2} \int_{0}^{1}|\mu-t| \\
& \times\left|f^{\prime}(t(a+\epsilon)+(1-t) b)\right| d t .
\end{aligned}
$$


4

The Scientific World Journal

Case $(q=1)$. By the convexity of $\left|f^{\prime}(x)\right|$ and Lemma 2, we have

$$
\begin{aligned}
& \int_{0}^{1}|1-\lambda-t|\left|f^{\prime}(t a+(1-t)(a+\epsilon))\right| d t \\
& =\int_{0}^{1}|1-\lambda-t| \\
& \quad \times\left|f^{\prime}\left(\left(1-\frac{\epsilon(1-t)}{b-a}\right) a+\frac{\epsilon(1-t)}{b-a} b\right)\right| d t \\
& \leq \int_{0}^{1}|1-\lambda-t|
\end{aligned}
$$

$\times\left[\left(1-\frac{\epsilon(1-t)}{b-a}\right)\left|f^{\prime}(a)\right|+\frac{\epsilon(1-t)}{b-a}\left|f^{\prime}(b)\right|\right] d t$

$=\left[\left(1-\frac{\epsilon}{b-a}\right)\left|f^{\prime}(a)\right|+\frac{\epsilon}{b-a}\left|f^{\prime}(b)\right|\right]$

$\times \int_{0}^{1}|1-\lambda-t| d t$

$+\left[\frac{\epsilon}{b-a}\left|f^{\prime}(a)\right|-\frac{\epsilon}{b-a}\left|f^{\prime}(b)\right|\right]$

$\times \int_{0}^{1} t|1-\lambda-t| d t$

$=\left[\left(1-\frac{\epsilon}{b-a}\right)\left|f^{\prime}(a)\right|+\frac{\epsilon}{b-a}\left|f^{\prime}(b)\right|\right]$

$\times \frac{(1-\lambda)^{2}+\lambda^{2}}{2}$

$+\left[\frac{\epsilon}{b-a}\left|f^{\prime}(a)\right|-\frac{\epsilon}{b-a}\left|f^{\prime}(b)\right|\right]$

$\times \frac{(1-\lambda)^{3}+(3-\lambda) \lambda^{2}}{6}$

$=\frac{1}{6}\left[3-6 \lambda+6 \lambda^{2}-\frac{\epsilon}{b-a}\left(2-3 \lambda+2 \lambda^{3}\right)\right]\left|f^{\prime}(a)\right|$

$+\frac{1}{6}\left[\frac{\epsilon}{b-a}\left(2-3 \lambda+2 \lambda^{3}\right)\right]\left|f^{\prime}(b)\right|$,

$\int_{0}^{1}|\mu-t|\left|f^{\prime}(t(a+\epsilon)+(1-t) b)\right| d t$

$=\int_{0}^{1}|\mu-t|$

$\times\left|f^{\prime}\left(\left(t-\frac{\epsilon t}{b-a}\right) a+\left(1-\left(t-\frac{\epsilon t}{b-a}\right)\right) b\right)\right| d t$

$\leq \int_{0}^{1}|\mu-t|\left[\left(t-\frac{\epsilon t}{b-a}\right)\left|f^{\prime}(a)\right|\right.$

$\left.+\left(1-\left(t-\frac{\epsilon t}{b-a}\right)\right)\left|f^{\prime}(b)\right|\right] d t$

$$
\begin{aligned}
= & {\left[\left(1-\frac{\epsilon}{b-a}\right)\left|f^{\prime}(a)\right|-\left(1-\frac{\epsilon}{b-a}\right)\left|f^{\prime}(b)\right|\right] } \\
& \times \int_{0}^{1} t|\mu-t| d t \\
& \quad+\left|f^{\prime}(b)\right| \int_{0}^{1}|\mu-t| d t \\
= & {\left[\left(1-\frac{\epsilon}{b-a}\right)\left|f^{\prime}(a)\right|-\left(1-\frac{\epsilon}{b-a}\right)\left|f^{\prime}(b)\right|\right] } \\
& \times \frac{\mu^{3}+(2+\mu)(1-\mu)^{2}}{6} \\
& +\left|f^{\prime}(b)\right| \frac{\mu^{2}+(1-\mu)^{2}}{2} \\
= & \frac{1}{6}\left[\left(1-\frac{\epsilon}{b-a}\right)\left(2-3 \mu+2 \mu^{3}\right)\right]\left|f^{\prime}(a)\right| \\
& +\frac{1}{6}\left[1-3 \mu+6 \mu^{2}-2 \mu^{3}+\frac{\epsilon}{b-a}\left(2-3 \mu+2 \mu^{3}\right)\right] \\
& \times\left|f^{\prime}(b)\right| \cdot
\end{aligned}
$$

Thus,

$$
\begin{aligned}
& \mid \epsilon \lambda f(a)+(b-a-\epsilon) \mu f(b) \\
& +[\epsilon(1-\lambda)+(b-a-\epsilon)(1-\mu)] \\
& \times f(a+\epsilon)-\int_{a}^{b} f(x) d x \mid \\
& \leq \frac{\epsilon^{2}}{6}\left[3-6 \lambda+6 \lambda^{2}-\frac{\epsilon}{b-a}\left(2-3 \lambda+2 \lambda^{3}\right)\right]\left|f^{\prime}(a)\right| \\
& +\frac{\epsilon^{2}}{6}\left[\frac{\epsilon}{b-a}\left(2-3 \lambda+2 \lambda^{3}\right)\right]\left|f^{\prime}(b)\right| \\
& +\frac{(b-a-\epsilon)^{2}}{6} \\
& \quad \times\left[\left(1-\frac{\epsilon}{b-a}\right)\left(2-3 \mu+2 \mu^{3}\right)\right]\left|f^{\prime}(a)\right| \\
& +\frac{(b-a-\epsilon)^{2}}{6} \\
& \quad \times\left[1-3 \mu+6 \mu^{2}-2 \mu^{3}+\frac{\epsilon}{b-a}\left(2-3 \mu+2 \mu^{3}\right)\right]\left|f^{\prime}(b)\right| .
\end{aligned}
$$

Case $(q>1)$. By Hölder's inequality, we have

$$
\begin{aligned}
& \mid \epsilon \lambda f(a)+(b-a-\epsilon) \mu f(b) \\
& \quad+[\epsilon(1-\lambda)+(b-a-\epsilon)(1-\mu)] \\
& \quad \times f(a+\epsilon)-\int_{a}^{b} f(x) d x \mid \leq \epsilon^{2}\left(\int_{0}^{1}|1-\lambda-t| d t\right)^{1-(1 / q)}
\end{aligned}
$$


The Scientific World Journal

5

$$
\begin{aligned}
& \times\left(\int_{0}^{1}|1-\lambda-t|\left|f^{\prime}(t a+(1-t)(a+\epsilon))\right|^{q} d t\right)^{1 / q} \\
& +(b-a-\epsilon)^{2}\left(\int_{0}^{1}|\mu-t| d t\right)^{1-(1 / q)} \\
& \times\left(\int_{0}^{1}|\mu-t|\left|f^{\prime}(t(a+\epsilon)+(1-t) b)\right|^{q} d t\right)^{1 / q} \\
& =\epsilon^{2}\left(\frac{(1-\lambda)^{2}+\lambda^{2}}{2}\right)^{1-(1 / q)} \\
& \times\left(\int_{0}^{1}|1-\lambda-t|\left|f^{\prime}(t a+(1-t)(a+\epsilon))\right|^{q} d t\right)^{1 / q} \\
& +(b-a-\epsilon)^{2}\left(\frac{\mu^{2}+(1-\mu)^{2}}{2}\right)^{1-(1 / q)} \\
& \times\left(\int_{0}^{1}|\mu-t|\left|f^{\prime}(t(a+\epsilon)+(1-t) b)\right|^{q} d t\right)^{1 / q} \cdot
\end{aligned}
$$

By the convexity of $\left|f^{\prime}(x)\right|^{q}$ and Lemma 2, we have

$$
\begin{aligned}
& \int_{0}^{1}|1-\lambda-t|\left|f^{\prime}(t a+(1-t)(a+\epsilon))\right|^{q} d t \\
&=\int_{0}^{1}|1-\lambda-t|\left|f^{\prime}\left(\left(1-\frac{\epsilon(1-t)}{b-a}\right) a+\frac{\epsilon(1-t)}{b-a} b\right)\right|^{q} d t \\
& \leq \int_{0}^{1}|1-\lambda-t| \\
& \quad \times\left[\left(1-\frac{\epsilon(1-t)}{b-a}\right)\left|f^{\prime}(a)\right|^{q}+\frac{\epsilon(1-t)}{b-a}\left|f^{\prime}(b)\right|^{q}\right] d t \\
&=\left[\left(1-\frac{\epsilon}{b-a}\right)\left|f^{\prime}(a)\right|^{q}+\frac{\epsilon}{b-a}\left|f^{\prime}(b)\right|^{q}\right] \int_{0}^{1}|1-\lambda-t| d t \\
&+ {\left[\frac{\epsilon}{b-a}\left|f^{\prime}(a)\right|^{q}-\frac{\epsilon}{b-a}\left|f^{\prime}(b)\right|^{q}\right] \int_{0}^{1} t|1-\lambda-t| d t } \\
&+\frac{1}{6}\left[\frac{\epsilon}{b-a}\left(2-3 \lambda+2 \lambda^{3}\right)\right]\left|f^{\prime}(b)\right|^{q}, \\
&= {\left[\left(1-\frac{\epsilon}{b-a}\right)\left|f^{\prime}(a)\right|^{q}+\frac{\epsilon}{b-a}\left|f^{\prime}(b)\right|^{q}\right] \frac{(1-\lambda)^{2}+\lambda^{2}}{2} } \\
&+\left[\frac{\epsilon}{b-a}\left|f^{\prime}(a)\right|^{q}-\frac{\epsilon}{b-a}\left|f^{\prime}(b)\right|^{q}\right] \frac{(1-\lambda)^{3}+(3-\lambda) \lambda^{2}}{6} \\
&= {\left[3-6 \lambda+6 \lambda^{2}-\frac{\epsilon}{b-a}\left(2-3 \lambda+2 \lambda^{3}\right)\right]\left|f^{\prime}(a)\right|^{q} }
\end{aligned}
$$

$$
\begin{aligned}
& \int_{0}^{1}|\mu-t|\left|f^{\prime}(t(a+\epsilon)+(1-t) b)\right|^{q} d t \\
& =\int_{0}^{1}|\mu-t|\left|f^{\prime}\left(\left(t-\frac{\epsilon t}{b-a}\right) a+\left(1-\left(t-\frac{\epsilon t}{b-a}\right)\right) b\right)\right|^{q} d t \\
& \leq \int_{0}^{1}|\mu-t| \\
& \quad \times\left[\left(t-\frac{\epsilon t}{b-a}\right)\left|f^{\prime}(a)\right|^{q}\right. \\
& =\left[\left(1-\frac{\epsilon}{b-a}\right)\left|f^{\prime}(a)\right|^{q}-\left(1-\frac{\epsilon}{b-a}\right)\left|f^{\prime}(b)\right|^{q}\right] \\
& \quad \times \int_{0}^{1} t|\mu-t| d t \\
& \left.\left.\left.\quad+\frac{\epsilon t}{b-a}\right)\right)\left|f^{\prime}(b)\right|^{q}\right] d t \\
& \quad \times\left|f^{\prime}(b)\right|^{q} \cdot(b)^{q}\left(\frac{\mu^{2}+(1-\mu)^{2}}{2}\right) \\
& \quad+\left|f^{\prime}(b)\right|^{q} \int_{0}^{1}|\mu-t| d t \\
& = \\
& \quad\left[\left(1-\frac{\epsilon}{b-a}\right)\left|f^{\prime}(a)\right|^{q}-\left(1-\frac{\epsilon}{b-a}\right)\left|f^{\prime}(b)\right|^{q}\right]
\end{aligned}
$$

Thus,

$$
\begin{aligned}
& \mid \epsilon \lambda f(a)+(b-a-\epsilon) \mu f(b) \\
& +[\epsilon(1-\lambda)+(b-a-\epsilon)(1-\mu)] \\
& \times f(a+\epsilon)-\int_{a}^{b} f(x) d x \mid \\
& \leq \epsilon^{2}\left(\frac{(1-\lambda)^{2}+\lambda^{2}}{2}\right)^{1-(1 / q)} \\
& \quad \times\left\{\frac{1}{6}\left[3-6 \lambda+6 \lambda^{2}-\frac{\epsilon}{b-a}\left(2-3 \lambda+2 \lambda^{3}\right)\right]\right. \\
& \left.\quad \times\left|f^{\prime}(a)\right|^{q}+\frac{1}{6}\left[\frac{\epsilon}{b-a}\left(2-3 \lambda+2 \lambda^{3}\right)\right]\left|f^{\prime}(b)\right|^{q}\right\}^{1 / q} \\
& +(b-a-\epsilon)^{2}\left(\frac{\mu^{2}+(1-\mu)^{2}}{2}\right)^{1-(1 / q)}
\end{aligned}
$$




$$
\begin{aligned}
\times\left\{\frac{1}{6}\right. & {\left[\left(1-\frac{\epsilon}{b-a}\right)\left(2-3 \mu+2 \mu^{3}\right)\right]\left|f^{\prime}(a)\right|^{q} } \\
+ & \frac{1}{6}\left[1-3 \mu+6 \mu^{2}-2 \mu^{3}+\frac{\epsilon}{b-a}\left(2-3 \mu+2 \mu^{3}\right)\right] \\
& \left.\times\left|f^{\prime}(b)\right|^{q}\right\}^{1 / q} .
\end{aligned}
$$

This proof is completed.

It is easy to notice that if we put $\epsilon=(b-a) / 2$ in Theorem 3 then we get the following.

Corollary 4 (see $[8]$ ). Let $\lambda, \mu \in[0,1]$ and let $f: I \subseteq \mathbb{R} \rightarrow$ $\mathbb{R}$ be differentiable on $I^{\circ}$ and $\{a, b\} \subseteq I$ with $a<b$. Assume that $f^{\prime} \in L[a, b]$. If $\left|f^{\prime}(x)\right|^{q}$ is convex on $[a, b]$ with $q \geq 1$, then

$$
\begin{aligned}
& \left|\frac{\lambda f(a)+\mu f(b)}{2}+\frac{2-\lambda-\mu}{2} f\left(\frac{a+b}{2}\right)-\frac{1}{b-a} \int_{a}^{b} f(x) d x\right| \\
& \leq \frac{b-a}{8}\left(1-2 \lambda+2 \lambda^{2}\right)^{1-1 / q} \\
& \quad \times\left[\left(\left(4-9 \lambda+12 \lambda^{2}-2 \lambda^{3}\right)\left|f^{\prime}(a)\right|^{q}\right.\right. \\
& \left.\left.\quad+\left(2-3 \lambda+2 \lambda^{3}\right)\left|f^{\prime}(b)\right|^{q}\right) \times(6)^{-1}\right]^{1 / q} \\
& +\frac{b-a}{8}\left(1-2 \mu+2 \mu^{2}\right)^{1-1 / q} \\
& \quad \times\left[\left(\left(2-3 \mu+2 \mu^{3}\right)\left|f^{\prime}(a)\right|^{q}\right.\right. \\
& \left.\left.\quad+\left(4-9 \mu+12 \mu^{2}-2 \mu^{3}\right)\left|f^{\prime}(b)\right|^{q}\right) \times(6)^{-1}\right]^{1 / q} .
\end{aligned}
$$

One can easily check that if we put $\epsilon=(b-a) / 3$ in Theorem 3, then we get the following.

Corollary 5. Let $\lambda, \mu \in[0,1]$ and let $f: I \subseteq \mathbb{R} \rightarrow \mathbb{R}$ be differentiable on $I^{\circ}$ and $\{a, b\} \subseteq I$ with $a<b$. Assume that $f^{\prime} \in L[a, b] . I f\left|f^{\prime}(x)\right|^{q}$ is convex on $[a, b]$ with $q \geq 1$, then

$$
\begin{aligned}
& \mid \frac{\lambda f(a)+2 \mu f(b)}{3}+\left(1-\frac{\lambda}{3}-\frac{2 \mu}{3}\right) f\left(\frac{2 a+b}{3}\right) \\
& -\frac{1}{b-a} \int_{a}^{b} f(x) d x \mid \\
& \leq \frac{b-a}{9}\left(\frac{(1-\lambda)^{2}+\lambda^{2}}{2}\right)^{1-(1 / q)} \\
& \quad \times\left(\frac{1}{18}\left(7-15 \lambda+18 \lambda^{2}-2 \lambda^{3}\right)\left|f^{\prime}(a)\right|^{q}\right. \\
& \left.\quad+\frac{1}{18}\left(2-3 \lambda+2 \lambda^{3}\right)\left|f^{\prime}(b)\right|^{q}\right)^{1 / q}
\end{aligned}
$$

$$
\begin{aligned}
& +\frac{4(b-a)}{9}\left(\frac{\mu^{2}+(1-\mu)^{2}}{2}\right)^{1-(1 / q)} \\
& \times\left(\frac{1}{9}\left(2-3 \mu+2 \mu^{3}\right)\left|f^{\prime}(a)\right|^{q}\right. \\
& \left.\quad+\frac{1}{18}\left(5-12 \mu+18 \mu^{2}-4 \mu^{3}\right)\left|f^{\prime}(b)\right|^{q}\right)^{1 / q} .
\end{aligned}
$$

One can easily check that if we put $\epsilon=2(b-a) / 3$ in Theorem 3 then we get the following.

Corollary 6. Let $\lambda, \mu \in[0,1]$ and let $f: I \subseteq \mathbb{R} \rightarrow \mathbb{R}$ be differentiable on $I^{\circ}$ and $\{a, b\} \subseteq I$ with $a<b$. Assume that $f^{\prime} \in L[a, b] . I f\left|f^{\prime}(x)\right|^{q}$ is convex on $[a, b]$ with $q \geq 1$, then

$$
\begin{aligned}
& \mid \frac{2 \lambda f(a)+\mu f(b)}{3}+\left(1-\frac{2 \lambda}{3}-\frac{\mu}{3}\right) f\left(\frac{a+2 b}{3}\right) \\
& -\frac{1}{b-a} \int_{a}^{b} f(x) d x \mid \\
& \leq \frac{4(b-a)}{9}\left(\frac{(1-\lambda)^{2}+\lambda^{2}}{2}\right)^{1-(1 / q)} \\
& \quad \times\left(\frac{1}{18}\left(5-12 \lambda+18 \lambda^{2}-4 \lambda^{3}\right)\left|f^{\prime}(a)\right|^{q}\right. \\
& \left.\quad+\frac{1}{9}\left(2-3 \lambda+2 \lambda^{3}\right)\left|f^{\prime}(b)\right|^{q}\right)^{1 / q} \\
& \left.+\frac{b-a}{9}\left(\frac{\mu^{2}+(1-\mu)^{2}}{2}\right)\right)^{1-(1 / q)} \\
& \quad \times\left(\frac{1}{18}\left(2-3 \mu+2 \mu^{3}\right)\left|f^{\prime}(a)\right|^{q}\right. \\
& \left.\quad+\frac{1}{18}\left(7-15 \mu+18 \mu^{2}-2 \mu^{3}\right)\left|f^{\prime}(b)\right|^{q}\right)^{1 / q} .
\end{aligned}
$$

It is easy to notice that if we put $\lambda=\mu=0$ in Theorem 3 then we get the following.

Corollary 7. Let $f: I \subseteq \mathbb{R} \rightarrow \mathbb{R}$ be differentiable on $I^{\circ}$ and $\{a, b\} \subseteq I$ with $a<b$. Assume that $f^{\prime} \in L[a, b]$ and $0<\epsilon<$ $b-a$. If $\left|f^{\prime}(x)\right|^{q}$ is convex on $[a, b]$ with $q \geq 1$, then

$$
\begin{aligned}
& 2^{1-(1 / q)}(b-a)\left|f(a+\epsilon)-\frac{1}{b-a} \int_{a}^{b} f(x) d x\right| \\
& \leq \epsilon^{2}\left(\left(\frac{1}{2}-\frac{\epsilon}{3(b-a)}\right)\left|f^{\prime}(a)\right|^{q}+\frac{\epsilon}{3(b-a)}\left|f^{\prime}(b)\right|^{q}\right)^{1 / q} \\
& +(b-a-\epsilon)^{2} \\
& \quad \times\left(\left(\frac{1}{3}-\frac{\epsilon}{3(b-a)}\right)\left|f^{\prime}(a)\right|^{q}\right. \\
& \left.\quad+\left(\frac{1}{6}+\frac{\epsilon}{3(b-a)}\right)\left|f^{\prime}(b)\right|^{q}\right)^{1 / q} .
\end{aligned}
$$


It is easy to notice that if we put $\lambda=\mu=1 / 2$ in Theorem 3 then we get the following.

Corollary 8. Let $f: I \subseteq \mathbb{R} \rightarrow \mathbb{R}$ be differentiable on $I^{\circ}$ and $\{a, b\} \subseteq I$ with $a<b$. Assume that $f^{\prime} \in L[a, b]$ and $0<\epsilon<b-a$. If $\left|f^{\prime}(x)\right|^{q}$ is convex on $[a, b]$ with $q \geq 1$, then

$$
\begin{aligned}
& 4^{1-(1 / q)} \mid \frac{\epsilon f(a)+(b-a-\epsilon) f(b)+(b-a) f(a+\epsilon)}{2} \\
& \quad-\int_{a}^{b} f(x) d x \mid \\
& \leq \epsilon^{2}\left(\left(\frac{1}{4}-\frac{\epsilon}{8(b-a)}\right)\left|f^{\prime}(a)\right|^{q}+\frac{\epsilon}{8(b-a)}\left|f^{\prime}(b)\right|^{q}\right)^{1 / q} \\
& +(b-a-\epsilon)^{2} \\
& \times\left(\left(\frac{1}{8}-\frac{\epsilon}{8(b-a)}\right)\left|f^{\prime}(a)\right|^{q}\right. \\
& \left.\quad+\left(\frac{1}{8}+\frac{\epsilon}{8(b-a)}\right)\left|f^{\prime}(b)\right|^{q}\right)^{1 / q} .
\end{aligned}
$$

It is easy to notice that if we put $\lambda=\mu=1$ in Theorem 3 then we get the following.

Corollary 9. Let $f: I \subseteq \mathbb{R} \rightarrow \mathbb{R}$ be differentiable on $I^{\circ}$ and $\{a, b\} \subseteq I$ with $a<b$. Assume that $f^{\prime} \in L[a, b]$ and $0<\epsilon<$ $b-a$. If $\left|f^{\prime}(x)\right|^{q}$ is convex on $[a, b]$ with $q \geq 1$, then

$$
\begin{aligned}
& 2^{1-(1 / q)}\left|\epsilon f(a)+(b-a-\epsilon) f(b)-\int_{a}^{b} f(x) d x\right| \\
& \leq \epsilon^{2}\left(\left(\frac{1}{3}-\frac{\epsilon}{6(b-a)}\right)\left|f^{\prime}(a)\right|^{q}+\frac{\epsilon}{6(b-a)}\left|f^{\prime}(b)\right|^{q}\right)^{1 / q} \\
& +(b-a-\epsilon)^{2} \\
& \quad \times\left(\left(\frac{1}{6}-\frac{\epsilon}{6(b-a)}\right)\left|f^{\prime}(a)\right|^{q}\right. \\
& \left.\quad+\left(\frac{1}{3}+\frac{\epsilon}{6(b-a)}\right)\left|f^{\prime}(b)\right|^{q}\right)^{1 / q} \cdot
\end{aligned}
$$

Theorem 10. Let $\lambda, \mu \in[0,1]$ and let $f: I \subseteq \mathbb{R} \rightarrow \mathbb{R}$ be differentiable on $I^{\circ}$ and $\{a, b\} \subseteq I$ with $a<b$. Assume that $f^{\prime} \in L[a, b]$ and $0<\epsilon<b-a$. If $\left|f^{\prime}(x)\right|^{q}$ is convex on $[a, b]$ with $q \geq 1$, then

$$
\begin{aligned}
& \mid \epsilon \lambda f(a)+(b-a-\epsilon) \mu f(b) \\
& \quad+[\epsilon(1-\lambda)+(b-a-\epsilon)(1-\mu)] \\
& \quad \times f(a+\epsilon)-\int_{a}^{b} f(x) d x \mid
\end{aligned}
$$

$$
\begin{aligned}
& \leq \epsilon^{2}\left\{\left[\frac{(1-\lambda)^{q+1}+\lambda^{q+1}}{q+1}-\left(\frac{\epsilon}{b-a}\right)\right.\right. \\
& \left.\quad \times \frac{(1+\lambda+q)(1-\lambda)^{q+1}+\lambda^{q+2}}{(q+1)(q+2)}\right]\left|f^{\prime}(a)\right|^{q} \\
& +\left[\left(\frac{\epsilon}{b-a}\right) \frac{(1+\lambda+q)(1-\lambda)^{q+1}+\lambda^{q+2}}{(q+1)(q+2)}\right] \\
& \left.\quad \times\left|f^{\prime}(b)\right|^{q}\right\}^{1 / q} \\
& +(b-a-\epsilon)^{2} \\
& \times\left\{\left[\left(1-\frac{\epsilon}{b-a}\right) \frac{\mu^{q+2}+(q+1+\mu)(1-\mu)^{q+1}}{(q+1)(q+2)}\right]\left|f^{\prime}(a)\right|^{q}\right. \\
& +\left[\frac{\mu^{q+1}+(1-\mu)^{q+1}}{q+1}-\left(1-\frac{\epsilon}{b-a}\right)\right. \\
& \left.\quad \times \frac{\mu^{q+2}+(q+1+\mu)(1-\mu)^{q+1}}{(q+1)(q+2)}\right] \\
& \left.\times\left|f^{\prime}(b)\right|^{q}\right\}^{1 / q} \cdot
\end{aligned}
$$

Proof. Suppose that $\left|f^{\prime}(x)\right|^{q}$ is convex on $[a, b]$ with $q \geq 1$. If $q=1$, then, by Theorem 3 , we have

$$
\begin{aligned}
\mid \epsilon \lambda f(a)+(b-a-\epsilon) \mu f(b) \\
+[\epsilon(1-\lambda)+(b-a-\epsilon)(1-\mu)] \\
\times f(a+\epsilon)-\int_{a}^{b} f(x) d x \mid \\
\leq \epsilon^{2}\left\{\frac{1}{6}\left[3-6 \lambda+6 \lambda^{2}-\frac{\epsilon}{b-a}\left(2-3 \lambda+2 \lambda^{3}\right)\right]\left|f^{\prime}(a)\right|\right. \\
\left.\quad+\frac{1}{6}\left[\frac{\epsilon}{b-a}\left(2-3 \lambda+2 \lambda^{3}\right)\right]\left|f^{\prime}(b)\right|\right\} \\
+(b-a-\epsilon)^{2} \\
\times\left\{\frac{1}{6}\left[\left(1-\frac{\epsilon}{b-a}\right)\left(2-3 \mu+2 \mu^{3}\right)\right]\left|f^{\prime}(a)\right|\right. \\
+\frac{1}{6}\left[1-3 \mu+6 \mu^{2}-2 \mu^{3}+\frac{\epsilon}{b-a}\left(2-3 \mu+2 \mu^{3}\right)\right] \\
\left.\quad \times\left|f^{\prime}(b)\right|\right\} \\
=\epsilon^{2}\left\{\left[\frac{(1-\lambda)^{2}+\lambda^{2}}{2}-\left(\frac{\epsilon}{b-a}\right) \frac{(2+\lambda)(1-\lambda)^{2}+\lambda^{3}}{6}\right]\right. \\
\quad \times\left|f^{\prime}(a)\right|+\left[\left(\frac{\epsilon}{b-a}\right) \frac{(2+\lambda)(1-\lambda)^{2}+\lambda^{3}}{6}\right] \\
\left.\times\left|f^{\prime}(b)\right|\right\}
\end{aligned}
$$


8

The Scientific World Journal

$$
\begin{aligned}
& +(b-a-\epsilon)^{2} \\
& \times\left\{\left[\left(1-\frac{\epsilon}{b-a}\right) \frac{\mu^{3}+(2+\mu)(1-\mu)^{2}}{6}\right]\left|f^{\prime}(a)\right|\right. \\
& +\left[\frac{\mu^{2}+(1-\mu)^{2}}{2}-\left(1-\frac{\epsilon}{b-a}\right)\right. \\
& \left.\left.\times \frac{\mu^{3}+(2+\mu)(1-\mu)^{2}}{6}\right]\left|f^{\prime}(b)\right|\right\} .
\end{aligned}
$$

Next, we suppose that $q>1$. By Lemma 1 and Hölder's inequality, we have

$$
\begin{aligned}
\mid \epsilon & \lambda f(a)+(b-a-\epsilon) \mu f(b) \\
& +[\epsilon(1-\lambda)+(b-a-\epsilon)(1-\mu)] \\
& \times f(a+\epsilon)-\int_{a}^{b} f(x) d x \mid \\
\leq & \epsilon^{2} \int_{0}^{1}|1-\lambda-t|\left|f^{\prime}(t a+(1-t)(a+\epsilon))\right| d t \\
& +(b-a-\epsilon)^{2} \int_{0}^{1}|\mu-t|\left|f^{\prime}(t(a+\epsilon)+(1-t) b)\right| d t \\
\leq & \epsilon^{2}\left(\int_{0}^{1} d t\right)^{1-(1 / q)} \\
& \times\left(\int_{0}^{1}|1-\lambda-t|^{q}\left|f^{\prime}(t a+(1-t)(a+\epsilon))\right|^{q} d t\right)^{1 / q} \\
& +(b-a-\epsilon)^{2} \\
& \left.+(b-a-\epsilon)^{2}\left(\int_{0}^{1} d t\right)^{1-(1 / q)}|\mu-t|^{q}\left|f^{\prime}(t(a+\epsilon)+(1-t) b)\right|^{q} d t\right)^{1 / q} \cdot \\
& \times\left(\int_{0}^{1}|\mu-t|^{q}\left|f^{\prime}(t(a+\epsilon)+(1-t) b)\right|^{q} d t\right)^{1 / q}
\end{aligned}
$$

By the convexity of $\left|f^{\prime}(x)\right|^{q}$ and Lemma 2, we have

$$
\begin{aligned}
& \int_{0}^{1}|1-\lambda-t|^{q}\left|f^{\prime}(t a+(1-t)(a+\epsilon))\right|^{q} d t \\
& =\int_{0}^{1}|1-\lambda-t|^{q}\left|f^{\prime}\left(\left(1-\frac{\epsilon(1-t)}{b-a}\right) a+\frac{\epsilon(1-t)}{b-a} b\right)\right|^{q} d t \\
& \leq \int_{0}^{1}|1-\lambda-t|^{q} \\
& \quad \times\left[\left(1-\frac{\epsilon(1-t)}{b-a}\right)\left|f^{\prime}(a)\right|^{q}+\frac{\epsilon(1-t)}{b-a}\left|f^{\prime}(b)\right|^{q}\right] d t
\end{aligned}
$$

$$
\begin{aligned}
& =\left[\left(1-\frac{\epsilon}{b-a}\right)\left|f^{\prime}(a)\right|^{q}+\frac{\epsilon}{b-a}\left|f^{\prime}(b)\right|^{q}\right] \\
& \quad \times \int_{0}^{1}|1-\lambda-t|^{q} d t \\
& \quad+\left[\frac{\epsilon}{b-a}\left|f^{\prime}(a)\right|^{q}-\frac{\epsilon}{b-a}\left|f^{\prime}(b)\right|^{q}\right] \\
& \quad \times \int_{0}^{1} t|1-\lambda-t|^{q} d t \\
& \quad=\left[\left(1-\frac{\epsilon}{b-a}\right)\left|f^{\prime}(a)\right|^{q}+\frac{\epsilon}{b-a}\left|f^{\prime}(b)\right|^{q}\right]
\end{aligned}
$$$$
\times \frac{(1-\lambda)^{q+1}+\lambda^{q+1}}{q+1}
$$$$
+\left[\frac{\epsilon}{b-a}\left|f^{\prime}(a)\right|^{q}-\frac{\epsilon}{b-a}\left|f^{\prime}(b)\right|^{q}\right]
$$$$
\times \frac{(1-\lambda)^{q+2}+(q+2-\lambda) \lambda^{q+1}}{(q+1)(q+2)}
$$$$
=\left[\frac{(1-\lambda)^{q+1}+\lambda^{q+1}}{q+1}-\left(\frac{\epsilon}{b-a}\right)\right.
$$$$
\begin{aligned}
& \left.\times \frac{(1+\lambda+q)(1-\lambda)^{q+1}+\lambda^{q+2}}{(q+1)(q+2)}\right]\left|f^{\prime}(a)\right|^{q} \\
+ & {\left[\left(\frac{\epsilon}{b-a}\right) \frac{(1+\lambda+q)(1-\lambda)^{q+1}+\lambda^{q+2}}{(q+1)(q+2)}\right]\left|f^{\prime}(b)\right|^{q}, }
\end{aligned}
$$$$
\int_{0}^{1}|\mu-t|^{q}\left|f^{\prime}(t(a+\epsilon)+(1-t) b)\right|^{q} d t
$$$$
=\int_{0}^{1}|\mu-t|^{q}
$$$$
\times\left|f^{\prime}\left(\left(t-\frac{\epsilon t}{b-a}\right) a+\left(1-\left(t-\frac{\epsilon t}{b-a}\right)\right) b\right)\right|^{q} d t
$$$$
\leq \int_{0}^{1}|\mu-t|^{q}
$$$$
\times\left[\left(t-\frac{\epsilon t}{b-a}\right)\left|f^{\prime}(a)\right|^{q}\right.
$$$$
\left.+\left(1-\left(t-\frac{\epsilon t}{b-a}\right)\right)\left|f^{\prime}(b)\right|^{q}\right] d t
$$$$
=\left[\left(1-\frac{\epsilon}{b-a}\right)\left|f^{\prime}(a)\right|^{q}-\left(1-\frac{\epsilon}{b-a}\right)\left|f^{\prime}(b)\right|^{q}\right]
$$$$
\times \int_{0}^{1} t|\mu-t|^{q} d t
$$

$$
+\left|f^{\prime}(b)\right|^{q} \int_{0}^{1}|\mu-t|^{q} d t
$$

$=\left[\left(1-\frac{\epsilon}{b-a}\right)\left|f^{\prime}(a)\right|^{q}-\left(1-\frac{\epsilon}{b-a}\right)\left|f^{\prime}(b)\right|^{q}\right]$

$\times \frac{\mu^{q+2}+(q+1+\mu)(1-\mu)^{q+1}}{(q+1)(q+2)}$

$+\left|f^{\prime}(b)\right|^{q}\left(\frac{\mu^{q+1}+(1-\mu)^{q+1}}{q+1}\right)$ 


$$
\begin{aligned}
& =\left[\left(1-\frac{\epsilon}{b-a}\right) \frac{\mu^{q+2}+(q+1+\mu)(1-\mu)^{q+1}}{(q+1)(q+2)}\right]\left|f^{\prime}(a)\right|^{q} \\
& +\left[\frac{\mu^{q+1}+(1-\mu)^{q+1}}{q+1}-\left(1-\frac{\epsilon}{b-a}\right)\right. \\
& \left.\quad \times \frac{\mu^{q+2}+(q+1+\mu)(1-\mu)^{q+1}}{(q+1)(q+2)}\right] \\
& \times\left|f^{\prime}(b)\right|^{q} .
\end{aligned}
$$

Thus,

$$
\left.\begin{array}{l}
\mid \epsilon \lambda f(a)+(b-a-\epsilon) \mu f(b) \\
+[\epsilon(1-\lambda)+(b-a-\epsilon)(1-\mu)] \\
\times f(a+\epsilon)-\int_{a}^{b} f(x) d x \mid \\
\leq \epsilon^{2}\left\{\left[\frac{(1-\lambda)^{q+1}+\lambda^{q+1}}{q+1}-\left(\frac{\epsilon}{b-a}\right)\right.\right. \\
\left.\quad \times \frac{(1+\lambda+q)(1-\lambda)^{q+1}+\lambda^{q+2}}{(q+1)(q+2)}\right]\left|f^{\prime}(a)\right|^{q} \\
+\left[\left(\frac{\epsilon}{b-a}\right) \frac{(1+\lambda+q)(1-\lambda)^{q+1}+\lambda^{q+2}}{(q+1)(q+2)}\right] \\
\left.\quad \times\left|f^{\prime}(b)\right|^{q}\right\}^{1 / q}+(b-a-\epsilon)^{2} \\
\times\left\{\left[\left(1-\frac{\epsilon}{b-a}\right) \frac{\mu^{q+2}+(q+1+\mu)(1-\mu)^{q+1}}{(q+1)(q+2)}\right]\right. \\
\quad \times\left|f^{\prime}(a)\right|^{q} \\
\quad\left[\frac{\mu^{q+1}+(1-\mu)^{q+1}}{q+1}-\left(1-\frac{\mu^{q+2}+(q+1+\mu)(1-\mu)^{q+1}}{b-a}\right)\right. \\
\quad(q+1)(q+2)
\end{array}\right]
$$

This proof is completed.
It is easy to notice that if we put $\epsilon=(b-a) / 2$ in Theorem 10 then we get the following.

Corollary 11 (see [8]). Let $\lambda, \mu \in[0,1]$ and let $f: I \subseteq \mathbb{R} \rightarrow$ $\mathbb{R}$ be differentiable on $I^{\circ}$ and $\{a, b\} \subseteq I$ with $a<b$. Assume that $f^{\prime} \in L[a, b]$. If $\left|f^{\prime}(x)\right|^{q}$ is convex on $[a, b]$ with $q \geq 1$, then

$$
\left|\frac{\lambda f(a)+\mu f(b)}{2}+\frac{2-\lambda-\mu}{2} f\left(\frac{a+b}{2}\right)-\frac{1}{b-a} \int_{a}^{b} f(x) d x\right|
$$

$$
\begin{aligned}
& \left.\leq \frac{b-a}{4}\left(\frac{1}{2(q+1)(q+2)}\right)\right)^{1 / q} \\
& \times\left[\left((q+3-\lambda)(1-\lambda)^{q+1}+(2 q+4-\lambda) \lambda^{q+1}\right)\left|f^{\prime}(a)\right|^{q}\right. \\
& \left.\quad+\left((q+1+\lambda)(1-\lambda)^{q+1}+\lambda^{q+2}\right)\left|f^{\prime}(b)\right|^{q}\right]^{1 / q} \\
& +\frac{b-a}{4}\left(\frac{1}{2(q+1)(q+2)}\right)^{1 / q} \\
& \times\left[\left((q+1+\mu)(1-\mu)^{q+1}+\mu^{q+2}\right)\left|f^{\prime}(a)\right|^{q}\right. \\
& \quad+\left((q+3-\mu)(1-\mu)^{q+1}+(2 q+4-\mu) \mu^{q+1}\right) \\
& \left.\quad \times\left|f^{\prime}(b)\right|^{q}\right]^{1 / q} .
\end{aligned}
$$

One can easily check that if we put $\epsilon=(b-a) / 3$ in Theorem 10 then we get the following.

Corollary 12. Let $\lambda, \mu \in[0,1]$ and let $f: I \subseteq \mathbb{R} \rightarrow \mathbb{R}$ be differentiable on $I^{\circ}$ and $\{a, b\} \subseteq I$ with $a<b$. Assume that $f^{\prime} \in L[a, b]$. If $\left|f^{\prime}(x)\right|^{q}$ is convex on $[a, b]$ with $q \geq 1$, then

$$
\begin{aligned}
& \mid \frac{\lambda f(a)+2 \mu f(b)}{3}+\left(1-\frac{\lambda}{3}-\frac{2 \mu}{3}\right) f\left(\frac{2 a+b}{3}\right) \\
& -\frac{1}{b-a} \int_{a}^{b} f(x) d x \mid \\
& \leq \frac{b-a}{9} \\
& \quad \times\left\{\frac{(5+2 q-\lambda)(1-\lambda)^{q+1}+(6+3 q-\lambda) \lambda^{q+1}}{3(q+1)(q+2)}\left|f^{\prime}(a)\right|^{q}\right. \\
& \left.\quad+\frac{(1+\lambda+q)(1-\lambda)^{q+1}+\lambda^{q+2}}{3(q+1)(q+2)}\left|f^{\prime}(b)\right|^{q}\right\}^{1 / q}
\end{aligned}
$$




$$
\begin{aligned}
& +\frac{4(b-a)}{9} \\
& \times\left\{\frac{2 \mu^{q+2}+2(q+1+\mu)(1-\mu)^{q+1}}{3(q+1)(q+2)}\left|f^{\prime}(a)\right|^{q}\right. \\
& +\frac{(6+3 q-2 \mu) \mu^{q+1}+(4+q-2 \mu)(1-\mu)^{q+1}}{3(q+1)(q+2)} \\
& \left.\quad \times\left|f^{\prime}(b)\right|^{q}\right\}^{1 / q} .
\end{aligned}
$$

One can easily check that if we put $\epsilon=2(b-a) / 3$ in Theorem 10 then we get the following.

Corollary 13. Let $\lambda, \mu \in[0,1]$ and let $f: I \subseteq \mathbb{R} \rightarrow \mathbb{R}$ be differentiable on $I^{\circ}$ and $\{a, b\} \subseteq I$ with $a<b$. Assume that $f^{\prime} \in L[a, b]$. If $\left|f^{\prime}(x)\right|^{q}$ is convex on $[a, b]$ with $q \geq 1$, then

$$
\begin{aligned}
& \frac{2 \lambda f(a)+\mu f(b)}{3}+\left(1-\frac{2 \lambda}{3}-\frac{\mu}{3}\right) f\left(\frac{a+2 b}{3}\right) \\
& -\frac{1}{b-a} \int_{a}^{b} f(x) d x \\
& \leq \frac{4(b-a)}{9} \\
& \times\left\{\frac{(4-q-2 \lambda)(1-\lambda)^{q+1}+(6+3 q-2 \lambda) \lambda^{q+1}}{3(q+1)(q+2)}\right. \\
& \times\left|f^{\prime}(a)\right|^{q}+\frac{2(1+\lambda+q)(1-\lambda)^{q+1}+2 \lambda^{q+2}}{3(q+1)(q+2)} \\
& \left.\times\left|f^{\prime}(b)\right|^{q}\right\}^{1 / q} \\
& +\frac{b-a}{9} \\
& \times\left\{\frac{\mu^{q+2}+(q+1+\mu)(1-\mu)^{q+1}}{3(q+1)(q+2)}\left|f^{\prime}(a)\right|^{q}\right. \\
& +\frac{(6+3 q-\mu) \mu^{q+1}+(5+2 q-\mu)(1-\mu)^{q+1}}{3(q+1)(q+2)} \\
& \left.\times\left|f^{\prime}(b)\right|^{q}\right\}^{1 / q}
\end{aligned}
$$

It is easy to notice that if we put $\lambda=\mu=0$ in Theorem 10 then we get the following.
Corollary 14. Let $f: I \subseteq \mathbb{R} \rightarrow \mathbb{R}$ be differentiable on $I^{\circ}$ and $\{a, b\} \subseteq I$ with $a<b$. Assume that $f^{\prime} \in L[a, b]$ and $0<\epsilon<$ $b-a$. If $\left|f^{\prime}(x)\right|^{q}$ is convex on $[a, b]$ with $q \geq 1$, then

$$
\begin{aligned}
& \left|(b-a) f(a+\epsilon)-\int_{a}^{b} f(x) d x\right| \\
& \leq \epsilon^{2}\left\{\left(\frac{1}{q+1}-\frac{\epsilon}{(b-a)(q+2)}\right)\left|f^{\prime}(a)\right|^{q}\right. \\
& \left.+\frac{\epsilon}{(b-a)(q+2)}\left|f^{\prime}(b)\right|^{q}\right\}^{1 / q} \\
& +(b-a-\epsilon)^{2} \\
& \times\left\{\left(1-\frac{\epsilon}{b-a}\right)\left(\frac{1}{q+2}\right)\left|f^{\prime}(a)\right|^{q}\right. \\
& \left.+\left(\frac{1}{q+1}-\left(1-\frac{\epsilon}{b-a}\right)\left(\frac{1}{q+2}\right)\right)\left|f^{\prime}(b)\right|^{q}\right\}^{1 / q} .
\end{aligned}
$$

It is easy to notice that if we put $\lambda=\mu=1 / 2$ in Theorem 10 then we get the following.

Corollary 15. Let $f: I \subseteq \mathbb{R} \rightarrow \mathbb{R}$ be differentiable on $I^{\circ}$ and $\{a, b\} \subseteq I$ with $a<b$. Assume that $f^{\prime} \in L[a, b]$ and $0<\epsilon<$ $b-a$. If $\left|f^{\prime}(x)\right|^{q}$ is convex on $[a, b]$ with $q \geq 1$, then

$$
\begin{aligned}
& \left|\frac{\epsilon f(a)+(b-a-\epsilon) f(b)+(b-a) f(a+\epsilon)}{2}-\int_{a}^{b} f(x) d x\right| \\
& \leq \frac{\epsilon^{2}}{2}\left(\frac{1}{q+1}\right)^{1 / q}\left\{\left(1-\frac{\epsilon}{2(b-a)}\right)\left|f^{\prime}(a)\right|^{q}\right. \\
& \left.+\frac{(b-a-\epsilon)^{2}}{2(b-a)}\left|f^{\prime}(b)\right|^{q}\right\}^{1 / q} \\
& \quad \times\left\{\left(\frac{1}{q+1}-\frac{\epsilon}{2(b-a)}\right)\left|f^{\prime}(a)\right|^{q}\right. \\
& \left.\quad+\left(\frac{1}{2}+\frac{\epsilon}{2(b-a)}\right)\left|f^{\prime}(b)\right|^{q}\right\}^{1 / q} .
\end{aligned}
$$

It is easy to notice that if we put $\lambda=\mu=1$ in Theorem 10 then we get the following. 
Corollary 16. Let $f: I \subseteq \mathbb{R} \rightarrow \mathbb{R}$ be differentiable on $I^{\circ}$ and $\{a, b\} \subseteq I$ with $a<b$. Assume that $f^{\prime} \in L[a, b]$ and $0<\epsilon<$ $b-a$. If $\left|f^{\prime}(x)\right|^{q}$ is convex on $[a, b]$ with $q \geq 1$, then

$$
\begin{aligned}
& \left|\epsilon f(a)+(b-a-\epsilon) f(b)-\int_{a}^{b} f(x) d x\right| \\
& \leq \epsilon^{2}\left(\frac{1}{q+1}\right)^{1 / q}\left\{\left(1-\frac{\epsilon}{(b-a)(q+2)}\right)\left|f^{\prime}(a)\right|^{q}\right. \\
& \left.+\frac{\epsilon}{(b-a)(q+2)}\left|f^{\prime}(b)\right|^{q}\right\}^{1 / q} \\
& +(b-a-\epsilon)^{2}\left(\frac{1}{q+1}\right)^{1 / q} \\
& \quad \times\left\{\left(1-\frac{\epsilon}{b-a}\right)\left(\frac{1}{q+2}\right)\left|f^{\prime}(a)\right|^{q}\right. \\
& \left.+\left(1-\left(1-\frac{\epsilon}{b-a}\right)\left(\frac{1}{q+2}\right)\right)\left|f^{\prime}(b)\right|^{q}\right\}^{1 / q} .
\end{aligned}
$$

\section{Applications}

In this section, we suppose that $\{s, q\} \subseteq[1, \infty)$ and $\left\{a, b, w_{a}, w_{b}\right\} \subseteq(0, \infty)$ with $a<b$. Let $0<\epsilon<b-a$.

The weighted arithmetic mean of data $\{a, b\}$ with weight $\left\{w_{a}, w_{b}\right\}$ is defined by

$$
A\left(a, b ; w_{a}, w_{b}\right)=\frac{w_{a} a+w_{b} b}{w_{a}+w_{b}} .
$$

The weighted geometric mean of data $\{a, b\}$ with weight $\left\{w_{a}, w_{b}\right\}$ is defined by

$$
G\left(a, b ; w_{a}, w_{b}\right)=a^{w_{a} /\left(w_{a}+w_{b}\right)} b^{w_{b} /\left(w_{a}+w_{b}\right)} .
$$
by

The generalized logarithmic mean of data $\{a, b\}$ is defined

$$
L(a, b)=\left(\frac{b^{s+1}-a^{s+1}}{(s+1)(b-a)}\right)^{1 / s} .
$$

The identric mean of data $\{a, b\}$ is defined by

$$
I(a, b)=\frac{1}{e}\left(\frac{b^{b}}{a^{a}}\right)^{1 /(b-a)} .
$$

Applying Corollary 7 with $f(x)=x^{s}$ on $(0, \infty)$, we get the following:

$$
\begin{aligned}
& \frac{2^{1-(1 / q)}(b-a)}{s}\left|A^{s}(a, b ; b-a-\epsilon, \epsilon)-L^{s}(a, b)\right| \\
& \leq \epsilon^{2}\left(\frac{a^{(s-1) q}}{2}+\frac{\epsilon\left(b^{(s-1) q}-a^{(s-1) q}\right)}{3(b-a)}\right)^{1 / q} \\
& +(b-a-\epsilon)^{2}\left(\frac{2 a^{(s-1) q}+b^{(s-1) q}}{6}+\frac{\epsilon\left(b^{(s-1) q}-a^{(s-1) q}\right)}{3(b-a)}\right)^{1 / q} .
\end{aligned}
$$

Applying Corollary 9 with $f(x)=x^{s}$ on $(0, \infty)$, we get the following:

$$
\begin{aligned}
& \frac{2^{1-(1 / q)}(b-a)}{s}\left|A\left(a^{s}, b^{s} ; \epsilon, b-a-\epsilon\right)-L^{s}(a, b)\right| \\
& \leq \epsilon^{2}\left(\frac{a^{(s-1) q}}{3}+\frac{\epsilon\left(b^{(s-1) q}-a^{(s-1) q}\right)}{6(b-a)}\right)^{1 / q} \\
& +(b-a-\epsilon)^{2}\left(\frac{a^{(s-1) q}+2 b^{(s-1) q}}{6}+\frac{\epsilon\left(b^{(s-1) q}-a^{(s-1) q}\right)}{6(b-a)}\right)^{1 / q} .
\end{aligned}
$$

Applying Corollary 7 with $f(x)=\ln x$ on $(0, \infty)$, we get the following:

$$
\begin{aligned}
2^{1-(1 / q)}(b-a)\left|\ln \left(\frac{A(a, b ; b-a-\epsilon, \epsilon)}{I(a, b)}\right)\right| \\
\leq \epsilon^{2}\left(\frac{a^{-q}}{2}+\frac{\epsilon\left(b^{-q}-a^{-q}\right)}{3(b-a)}\right)^{1 / q} \\
\quad+(b-a-\epsilon)^{2}\left(\frac{2 a^{-q}+b^{-q}}{6}+\frac{\epsilon\left(b^{-q}-a^{-q}\right)}{3(b-a)}\right)^{1 / q} .
\end{aligned}
$$

Applying Corollary 9 with $f(x)=\ln x$ on $(0, \infty)$, we get the following:

$$
\begin{aligned}
2^{1-(1 / q)}(b-a)\left|\ln \left(\frac{G(a, b ; \epsilon, b-a-\epsilon)}{I(a, b)}\right)\right| \\
\leq \epsilon^{2}\left(\frac{a^{-q}}{3}+\frac{\epsilon\left(b^{-q}-a^{-q}\right)}{6(b-a)}\right)^{1 / q} \\
\quad+(b-a-\epsilon)^{2}\left(\frac{a^{-q}+2 b^{-q}}{6}+\frac{\epsilon\left(b^{-q}-a^{-q}\right)}{6(b-a)}\right)^{1 / q} .
\end{aligned}
$$

Applying Corollary 14 with $f(x)=x^{s}$ on $(0, \infty)$, we get the following:

$$
\begin{aligned}
& \frac{b-a}{s}\left|A^{s}(a, b ; b-a-\epsilon, \epsilon)-L^{s}(a, b)\right| \\
& \leq \epsilon^{2}\left(\frac{a^{(s-1) q}}{q+1}+\frac{\epsilon\left(b^{(s-1) q}-a^{(s-1) q}\right)}{(b-a)(q+2)}\right)^{1 / q} \\
& +(b-a-\epsilon)^{2} \\
& \quad \times\left(\frac{b^{(s-1) q}}{q+1}+\frac{a^{(s-1) q}-b^{(s-1) q}}{q+2}+\frac{\epsilon\left(b^{(s-1) q}-a^{(s-1) q}\right)}{(b-a)(q+2)}\right)^{1 / q} .
\end{aligned}
$$


Applying Corollary 16 with $f(x)=x^{s}$ on $(0, \infty)$, we get the following:

$$
\begin{aligned}
& \frac{(q+1)^{1 / q}(b-a)}{s}\left|A\left(a^{s}, b^{s} ; \epsilon, b-a-\epsilon\right)-L^{s}(a, b)\right| \\
& \leq \epsilon^{2}\left(a^{(s-1) q}+\frac{\epsilon\left(b^{(s-1) q}-a^{(s-1) q}\right)}{(b-a)(q+2)}\right)^{1 / q} \\
& +(b-a-\epsilon)^{2} \\
& \quad \times\left(b^{(s-1) q}+\frac{a^{(s-1) q}-b^{(s-1) q}}{q+2}+\frac{\epsilon\left(b^{(s-1) q}-a^{(s-1) q}\right)}{(b-a)(q+2)}\right)^{1 / q} .
\end{aligned}
$$

Applying Corollary 14 with $f(x)=\ln x$ on $(0, \infty)$, we get the following:

$$
\begin{aligned}
& (b-a)\left|\ln \left(\frac{A(a, b ; b-a-\epsilon, \epsilon)}{I(a, b)}\right)\right| \\
& \leq \epsilon^{2}\left(\frac{a^{-q}}{q+1}+\frac{\epsilon\left(b^{-q}-a^{-q}\right)}{(b-a)(q+2)}\right)^{1 / q} \\
& +(b-a-\epsilon)^{2}\left(\frac{b^{-q}}{q+1}+\frac{a^{-q}-b^{-q}}{q+2}+\frac{\epsilon\left(b^{-q}-a^{-q}\right)}{(b-a)(q+2)}\right)^{1 / q} .
\end{aligned}
$$

Applying Corollary 16 with $f(x)=\ln x$ on $(0, \infty)$, we get the following:

$$
\begin{aligned}
& (q+1)^{1 / q}(b-a)\left|\ln \left(\frac{G(a, b ; \epsilon, b-a-\epsilon)}{I(a, b)}\right)\right| \\
& \leq \epsilon^{2}\left(a^{-q}+\frac{\epsilon\left(b^{-q}-a^{-q}\right)}{(b-a)(q+2)}\right)^{1 / q} \\
& +(b-a-\epsilon)^{2}\left(b^{-q}+\frac{a^{-q}-b^{-q}}{q+2}+\frac{\epsilon\left(b^{-q}-a^{-q}\right)}{(b-a)(q+2)}\right)^{1 / q} .
\end{aligned}
$$

\section{Conflict of Interests}

The author declares that there is no conflict of interests regarding the publication of this paper.

\section{Acknowledgment}

The author would like to thank the referees for their useful comments and suggestions.

\section{References}

[1] M. Alomari and M. Darus, "On the Hadamard's inequality for log-convex functions on the coordinates," Journal of Inequalities and Applications, vol. 2009, Article ID 283147, 13 pages, 2009.
[2] C. Dinu, "Hermite-Hadamard inequality on time scales," Journal of Inequalities and Applications, vol. 2008, Article ID 287947, 24 pages, 2008.

[3] D. S. Mitrinović, J. E. Pečarić, and A. M. Fink, Classical and New Inequalities in Analysis, vol. 61 of Mathematics and Its Applications, Kluwer Academic Publishers, Dordrecht, The Netherlands, 1993.

[4] S. S. Dragomir and R. P. Agarwal, "Two inequalities for differentiable mappings and applications to special means of real numbers and to trapezoidal formula," Applied Mathematics Letters, vol. 11, no. 5, pp. 91-95, 1998.

[5] C. E. M. Pearce and J. Pečarić, "Inequalities for differentiable mappings with application to special means and quadrature formulae," Applied Mathematics Letters, vol. 13, no. 2, pp. 51-55, 2000.

[6] U. S. Kirmaci, "Inequalities for differentiable mappings and applications to special means of real numbers and to midpoint formula," Applied Mathematics and Computation, vol. 147, no. 1, pp. 137-146, 2004.

[7] M. Z. Sarikaya, E. Set, and M. E. Özdemir, "On new inequalities of Simpson's type for convex functions," RGMIA Research Report Collection, vol. 13, no. 2, article 2, 8 pages, 2010.

[8] B. Y. Xi and F. Qi, "Some integral inequalities of HermiteHadamard type for convex functions with applications to means," Journal of Function Spaces and Applications, vol. 2012, Article ID 980438, 14 pages, 2012.

[9] R. F. Bai and F. Qi, "Some inequalities of Hermite-Hadamard type for $h$-convex functions," Advances in Inequalities and Applications, vol. 2, no. 1, pp. 1-15, 2013.

[10] R. F. Bai, F. Qi, and B. Y. Xi, "Hermite-Hadamard type inequalities for $m$ - and $(\alpha, m)$-logarithmically convex functions," Filomat, vol. 27, no. 1, pp. 1-7, 2013.

[11] S. P. Bai, S. H. Wang, and F. Qi, "Some Hermite-Hadamard type inequalities for $n$-time differentiable $(\alpha, m)$-convex functions," Journal of Inequalities and Applications, vol. 2012, article 267, 11 pages, 2012.

[12] A. Barani, S. Barani, and S. S. Dragomir, "Refinements of Hermite-Hadamard inequalities for functions when a power of the absolute value of the second derivative is P-convex," Journal of Applied Mathematics, vol. 2012, Article ID 615737, 10 pages, 2012.

[13] L. Chun and F. Qi, "Integral inequalities of Hermite-Hadamard type for functions whose $3 \mathrm{rd}$ derivatives are $s$-convex," Applied Mathematics, vol. 3, no. 11, pp. 1680-1685, 2012.

[14] M. E. Özdemir, M. Gürbüz, and H. Kavurmaci, "HermiteHadamard-type inequalities for $(g, \varphi h)$-convex dominated functions," Journal of Inequalities and Applications, vol. 2013, article 184, 7 pages, 2013.

[15] J. Park, "Hermite-Hadamard and Simpson-like type inequalities for differentiable $(\alpha, m)$-convex mappings," International Journal of Mathematics and Mathematical Sciences, vol. 2012, Article ID 809689, 12 pages, 2012.

[16] F. Qi, Z. L. Wei, and Q. Yang, "Generalizations and refinements of Hermite-Hadamard's inequality," The Rocky Mountain Journal of Mathematics, vol. 35, no. 1, pp. 235-251, 2005.

[17] M. Z. Sarikaya and H. Yaldiz, "On Hermite Hadamard-type inequalities for strongly log-convex functions," International Journal of Modern Mathematical Sciences, vol. 6, no. 1, pp. 1-8, 2013.

[18] E. Set, M. E. Özdemir, and S. S. Dragomir, "On the HermiteHadamard inequality and other integral inequalities involving 
two functions," Journal of Inequalities and Applications, vol. 2010, Article ID 148102, 9 pages, 2010.

[19] B. Sroysang, "On the Hermite-Hadamard inequality and other integral inequalities involving several functions," Journal of Function Spaces and Applications, vol. 2013, Article ID 921828, 6 pages, 2013.

[20] S. H. Wang, B. Y. Xi, and F. Qi, "On Hermite-Hadamard type inequalities for $(\alpha, m)$-convex functions," International Journal of Open Problems in Computer Science and Mathematics, vol. 5, no. 4, pp. 47-56, 2012.

[21] B. Y. Xi, R. F. Bai, and F. Qi, "Hermite-Hadamard type inequalities for $m$ - and $(\alpha, m)$-geometrically convex functions," Aequationes Mathematicae, vol. 84, no. 3, pp. 261-269, 2012.

[22] B. Y. Xi, S. H. Wang, and F. Qi, "Some inequalities of HermiteHadamard type for functions whose 3rd derivatives are $P$ convex," Applied Mathematics, vol. 3, no. 12, pp. 1898-1902, 2012.

[23] T. Y. Zhang, A. P. Ji, and F. Qi, "On integral inequalities of Hermite-Hadamard type for $s$-geometrically convex functions," Abstract and Applied Analysis, vol. 2012, Article ID 560586, 14 pages, 2012. 


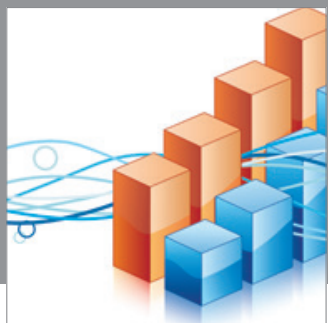

Advances in

Operations Research

mansans

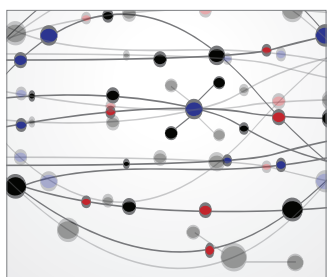

The Scientific World Journal
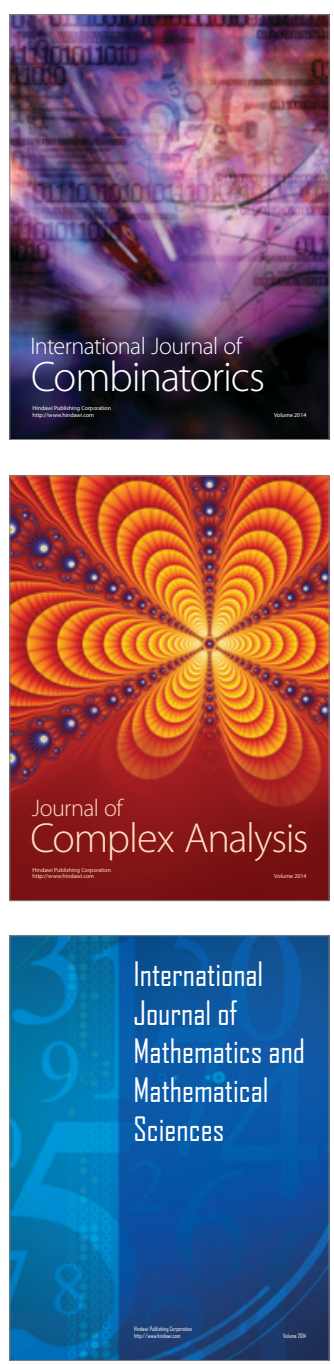
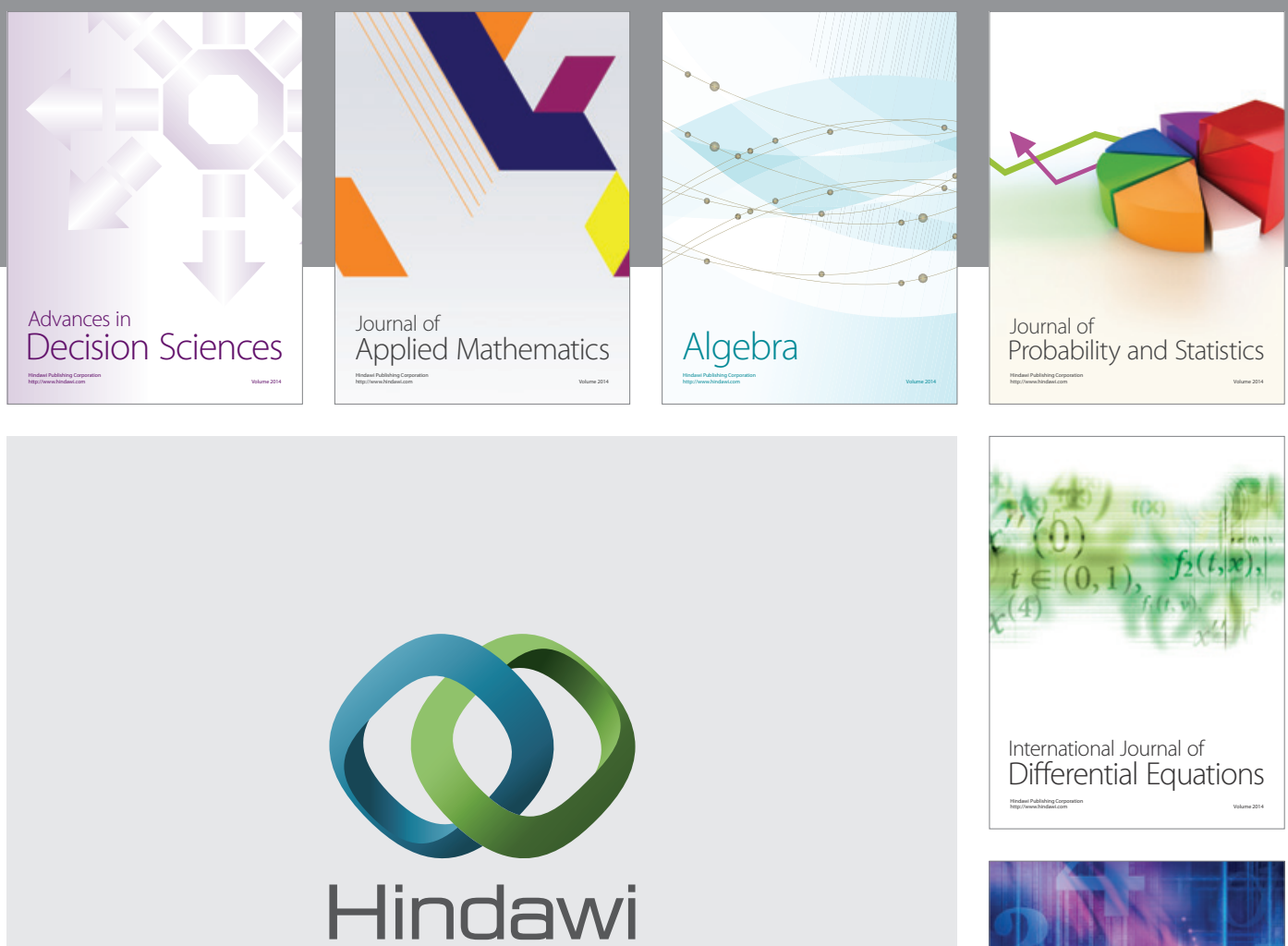

Submit your manuscripts at http://www.hindawi.com
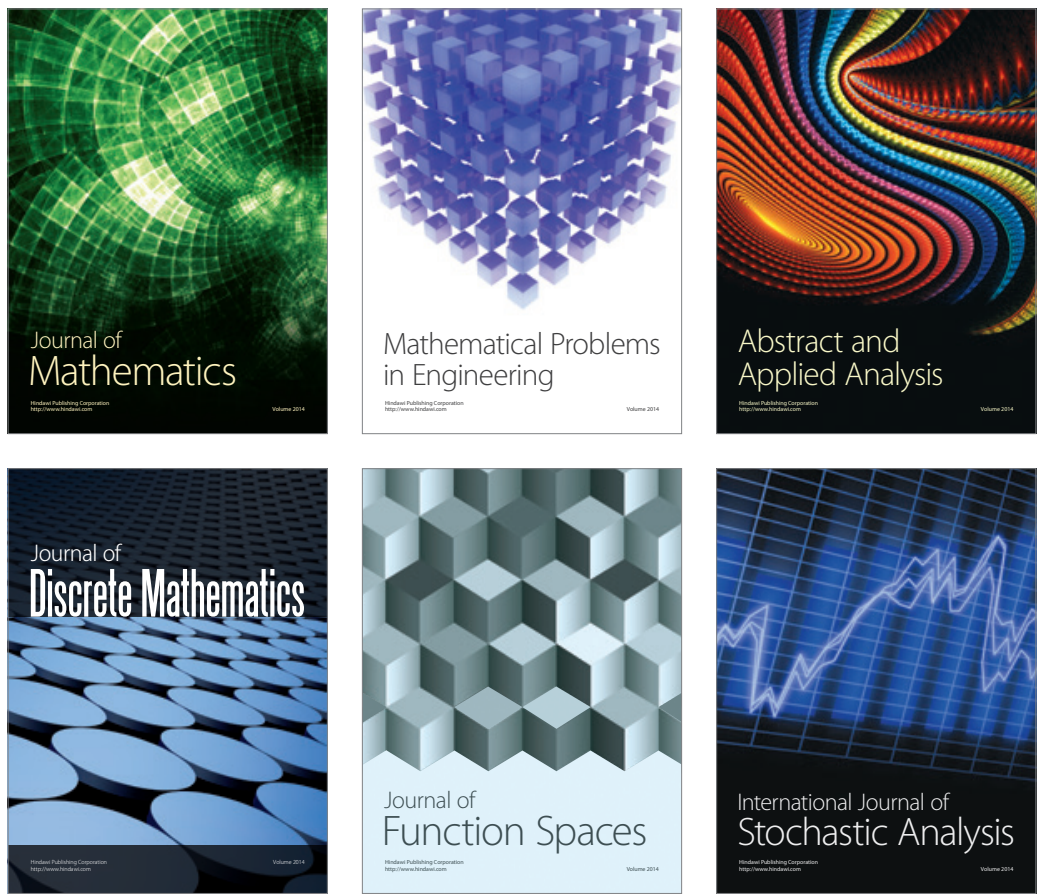

Journal of

Function Spaces

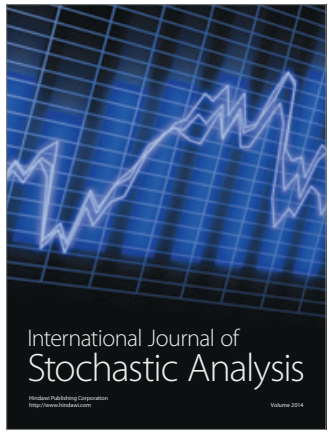

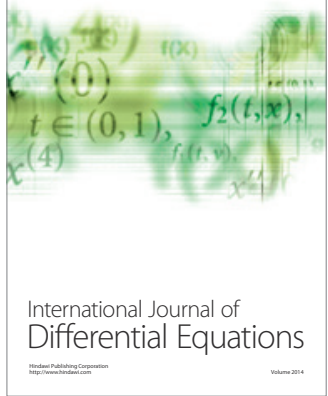
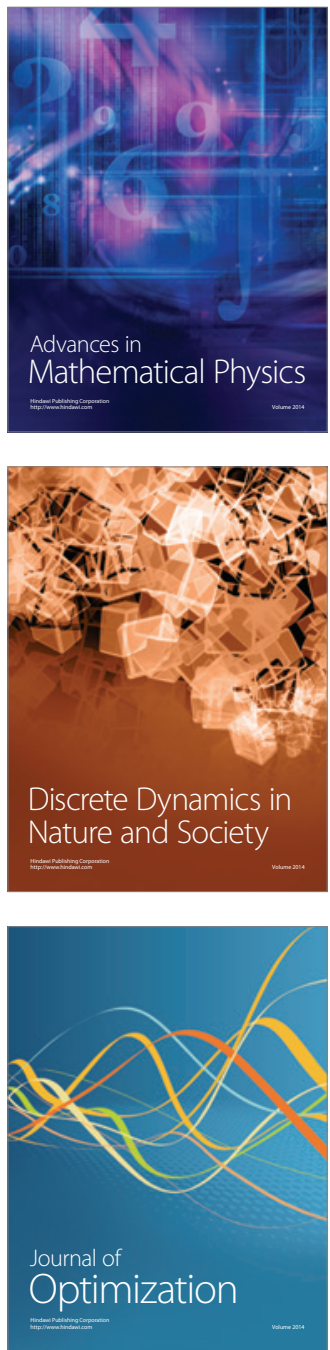